\title{
Retinoic Acid-Related Orphan Receptor Alpha Reprograms Glucose Metabolism in Glutamine-Deficient Hepatoma Cells
}

\author{
Jun-Kyu Byun, ${ }^{1 *}$ Yeon-Kyung Choi, ${ }^{1,2 *}$ Yu Na Kang, ${ }^{3}$ Byoung Kuk Jang, ${ }^{4}$ Koo Jeong Kang, ${ }^{5}$ \\ Yong Hyun Jeon, ${ }^{1,6}$ Ho-Won Lee, ${ }^{1,6}$ Jae-Han Jeon, ${ }^{1,2}$ Seung-Hoi Koo, ${ }^{7}$ Won-Il Jeong, ${ }^{8}$ Robert A. Harris, ${ }^{9}$ \\ In-Kyu Lee, ${ }^{1,2,10}$ and Keun-Gyu Park ${ }^{1,2}$
}

The metabolism of glutamine and glucose is recognized as a promising therapeutic target for the treatment of cancer; however, targeted molecules that mediate glutamine and glucose metabolism in cancer cells have not been addressed. Here, we show that restricting the supply of glutamine in hepatoma cells, including HepG 2 and Hep3B cells, markedly increased the expression of retinoic acid-related orphan receptor alpha (ROR $\alpha)$. Up-regulation of ROR $\alpha$ in glutaminedeficient hepatoma cells resulted from an increase in the level of cellular reactive oxygen species and in the nicotinamide adenine dinucleotide phosphate/nicotinamide adenine dinucleotide phosphate reduced (NADP ${ }^{+} / \mathrm{NADPH}$ ) ratio, which was consistent with a reduction in the glutathione/glutathione disulfide (GSH/GSSG) ratio. Adenovirus (Ad)-mediated overexpression of $\operatorname{ROR} \alpha(\operatorname{Ad}-\mathrm{ROR} \alpha)$ or treatment with the ROR $\alpha$ activator, SR1078, reduced aerobic glycolysis and down-regulated biosynthetic pathways in hepatoma cells. Ad-ROR $\alpha$ and SR1078 reduced the expression of pyruvate dehydrogenase kinase 2 (PDK2) and inhibited the phosphorylation of pyruvate dehydrogenase and subsequently shifted pyruvate to complete oxidation. The ROR $\alpha$-mediated decrease in PDK2 levels was caused by up-regulation of $\mathrm{p} 21$, rather than $\mathrm{p} 53$. Furthermore, $\mathrm{ROR} \alpha$ inhibited hepatoma growth both in vitro and in a xenograft model in vivo. We also found that suppression of PDK2 inhibited hepatoma growth in a xenograft model. These findings mimic the altered glucose utilization and hepatoma growth caused by glutamine deprivation. Finally, tumor tissue from 187 hepatocellular carcinoma patients expressed lower levels of $\operatorname{ROR} \alpha$ than adjacent nontumor tissue, supporting a potential beneficial effect of ROR $\alpha$ activation in the treatment of liver cancer. Conclusion: ROR $\alpha$ mediates reprogramming of glucose metabolism in hepatoma cells in response to glutamine deficiency. The relationships established here between glutamine metabolism, ROR $\alpha$ expression and signaling, and aerobic glycolysis have implications for therapeutic targeting of liver cancer metabolism. (HEPATOLOGY 2015;61:953-964)

$\mathrm{L}$ iver cancer is one of the most common and fatal malignancies. ${ }^{1}$ The most prevalent types of liver adults and hepatoblastoma $(\mathrm{HB})$ in children. ${ }^{2}$ Curative cancer are hepatocellular carcinoma (HCC) in therapies are effective only if HCC is diagnosed in its early stages; however, most patients present with intermediate or

Abbreviations: Ad, adenovirus; AOA, aminooxyacetate; ATP, adenosine triphosphate; BPTES, bis-2-(5-phenylacetamido-1,2,4-thiadiazol-2-yl)ethyl sulfide; FBS, fetal bovine serum; DMSO, dimethyl sulfoxide; G6PD, glucose-6-phosphate dehydrogenase; GLS, glutaminase; GPD1, glycerol-3-phosphate dehydrogenase 1; GSH, glutathione; GSSG, glutathione disulfide; HB, hepatoblastoma; HCC, hepatocellular carcinoma; IHC, immunohistochemical; IRB, institutional review board; LDHA, lactate dehydrogenase A; mRNA, messenger RNA; NADP, nicotinamide adenine dinucleotide phosphate; NADPH, nicotinamide adenine dinucleotide phosphate reduced; OXPHOS, oxidative phosphorylation; PDH, pyruvate dehydrogenase; PDK, pyruvate dehydrogenase kinase; PEP, phosphoenolpyruvate; PHGDH, phosphoglycerate dehydrogenase; PKM2, pyruvate kinase M2; pRb, retinoblastoma protein; qRT-PCR, quantitative reverse-transcriptase polymerase chain reaction; $R O R \alpha$, retinoic acid-related orphan receptor alpha; ROS, reactive oxygen species; SC, subcutaneously; SEM, standard error of the mean; sh, short hairpin; siRNA, small interfering RNA; TCA, tricarboxylic acid; TNM, tumor node metastasis; TXNIP, thioredoxin-interacting protein.

From the ${ }^{1}$ Leading-edge Research Center for Drug Discovery and Development for Diabetes and Metabolic Disease, Kynngpook National University Hospital, Daegu, South Korea; ${ }^{2}$ Department of Internal Medicine, Kynngpook National University School of Medicine, Daegu, South Korea; Departments of ${ }^{3}$ Pathology, ${ }^{4}$ Internal Medicine, and ${ }^{5}$ Surgery, Keimyung University School of Medicine, Daegu, South Korea; ${ }^{6}$ Department of Nuclear Medicine, Kyungpook National University School of Medicine, Daegu, South Korea; ${ }^{7}$ Department of Life Sciences, Korea University, Seoul, South Korea; ${ }^{8}$ Laboratory of Liver Research, Graduate School of Medical Science and Engineering, KAIST, Daejeon, Republic of Korea; ${ }^{9}$ Roudebush VA Medical Center and the Department of Biochemistry and Molecular Biology, Indiana University School of Medicine, Indianapolis, IN; and ${ }^{10}$ BK21 Plus KNU Biomedical Convergence Program, Kyungpook National University School of Medicine, Daegu, South Korea. 
advanced disease. ${ }^{3}$ Although many oncogenes and mutated tumor-suppressor genes have been identified and targeted in potential therapeutic strategies, sorafenib, a multikinase inhibitor, is the only drug approved for patients with advanced HCC. ${ }^{4}$ However, this drug is beneficial in only approximately $30 \%$ of patients and acquired resistance often develops within 6 months. ${ }^{5}$ On the other hand, although combination chemotherapy for $\mathrm{HB}$ showed a higher response rate than HCC, a considerable percentage of patients with $\mathrm{HB}$ did not respond to chemotherapy. ${ }^{6}$ Alternative approaches, such as developing new drugs for modulating cancer metabolism, are therefore required to overcome these barriers and enhance therapeutic efficacy.

Recently, there has been increasing evidence suggesting that the reprogramming of tumor metabolism using novel therapeutic targets could be an effective anticancer strategy. ${ }^{7}$ As is the case for other types of proliferating cancer cells, glutamine and glucose are essential nutrients for HCC growth and survival. ${ }^{8} \mathrm{~A}$ high rate of aerobic glycolysis, known as the Warburg effect, is a hallmark of cancer cell glucose metabolism. The aerobic glycolytic pathway generates adenosine triphosphate (ATP) and produces metabolic intermediates required for cancer cell proliferation. ${ }^{9}$ Glutamine replenishes a truncated tricarboxylic acid (TCA) cycle and provides essential intermediates for intracellular nucleotide and nonessential amino acid biosynthesis. ${ }^{10}$ Although proliferating cancer cells elicit a pronounced Warburg effect, their TCA cycle remains intact and becomes more dependent on glutamine metabolism. ${ }^{11}$ Glutamine also plays a critical role in alleviating levels of reactive oxygen species (ROS) as it donates carbon and nitrogen for the production of glutathione (GSH), a major intracellular antioxidant, and is a substrate for the production of reducing equivalents, such as nicotinamide adenine dinucleotide phosphate reduced (NADPH). ${ }^{12}$

The rationale for therapies directed against glutamine metabolism in cancer comes, at least in part, from the observation that glutamine deprivation inhibits cancer cell growth. ${ }^{13}$ Nevertheless, the efficacy and toxicity of pharmacological inhibitors of glutamine metabolism remain largely unknown in cancer patients. However, systemic blocking of glutamine metabolism may cause serious side effects because glutamine consumption occurs largely in the gut and kidney. During acidosis, the kidneys substantially increase consumption of glutamine to maintain the acid-base balance. ${ }^{14}$ Therefore, it would be valuable to develop a strategy that mimics glutamine depletion in tumor tissue without negatively affecting glutamine metabolism in normal tissue.

A growing body of evidence suggests that several orphan nuclear receptors are amplified in, and prognostic for, some human cancers, ${ }^{15}$ and that some are involved in regulation of nutrient metabolism. ${ }^{16}$ Cancer-associated orphan nuclear receptors that alter glucose metabolism in response to glutamine deprivation could provide selective targets for cancer therapy. The retinoic acid receptor-related orphan receptor alpha $(\mathrm{ROR} \alpha)$ is a potent regulator of normal physiological functions, such as lipid and steroid metabolism. ${ }^{17}$ A recent study demonstrated that $\operatorname{ROR} \alpha$ could functionally interact with potent oncogenic systems, such as the Wnt- and protein kinase C-signaling pathways and tumor-suppressor p53 stabilization, ${ }^{18,19}$ indicating its involvement in the regulation of cell growth and tumorigenesis. However, whether $\operatorname{ROR} \alpha$ regulates glucose and glutamine metabolism in hepatoma cells and the mechanisms responsible for glutamine deprivation-mediated inhibition of hepatoma cell growth remain largely unknown. The aim of this study was to determine whether $\operatorname{ROR} \alpha$ mediates the alteration of glucose metabolism and reduction in growth observed in hepatoma cells in response to glutamine depletion and whether modulation of $\operatorname{ROR} \alpha$ activity has potential as a therapeutic strategy for liver cancer, including HCC and HB.

Received May 8, 2014; accepted October 17, 2014.

Additional Supporting Information may be found at onlinelibrary. wiley.com/doi/10.1002/hep.27577/suppinfo.

This work was supported by grants from the National Research Foundation of Korea (2012R1A2A2A01043867 and 2012R1A2A1A03670452), funded by the Ministry of Science, ICT, and Future Planning, and a grant from the Korea Health Technology ReD D Project, Ministry of Health and Welfare, Republic of Korea (A111345).

${ }^{*}$ These authors contributed equally.

Address reprint requests to: Keun-Gyu Park, M.D., Ph.D., Department of Internal Medicine, Kyungpook National University School of Medicine, 130 Dongdeok-ro, Jung-gu, Daegu 700-721, South Korea. E-mail: kpark@knu.ac.kr; fax: +82-53-426-6722 or In-Kyu Lee, M.D., Ph.D., Department of Internal Medicine, Kyungpook National University School of Medicine, 130 Dongdeok-ro, Jung-gu, Daegu 700-721, South Korea. E-mail: leei@knu.ac.kr; fax: +82-53420-2046.

Copyright $@ 2014$ by the American Association for the Study of Liver Diseases.

View this article online at wileyonlinelibrary.com.

DOI 10.1002/hep.27577

Potential conflict on interest: Nothing to report. 


\section{Materials and Methods}

Patients and Specimens. For details on patients and specimens, see the Materials and Methods section of the Supporting Information.

Cells. Hepatoma cell lines (HepG2, Hep3B, and Huh7 cells) were maintained in RPMI 1640 medium containing $10 \mathrm{mM}$ of D-glucose and $2 \mathrm{mM}$ of L-glutamine supplemented with $10 \%$ fetal bovine serum (FBS) and 1\% penicillin/streptomycin. THLE-2 cells, a human normal liver cell line, was maintained in BEBM supplemented with the BEGM Bullet Kit and 10\% FBS according to American Type Culture Collection guidelines. For glutamine deprivation experiments, cells were incubated in glutamine-free RPMI supplemented with $10 \%$ dialyzed FBS. Cell number was calculated using a hemocytometer.

For details on western blotting analysis, glucose uptake measurements, and quantitative reversetranscriptase polymerase chain reaction (qRT-PCR), see the Materials and Methods section of the Supporting Information.

Pyruvate Dehydrogenase Activity Measurement. The activity of pyruvate dehydrogenase (PDH) in cell extracts was measured and calculated using a $\mathrm{PDH}$ Activity Assay Kit (Novagen; Merck KGaA, Darmstadt, Germany), according to the manufacturer's instructions.

GSH Concentration Measurement. For details on GSH concentration measurement, see the Materials and Methods section of the Supporting Information.

NADP ${ }^{+} / N A D P H$ Measurement. The intracellular $\mathrm{NADP}^{+} / \mathrm{NADPH}$ ratio in HepG2 and Hep3B cells after 24 hours cultured in glutamine-free RPMI was assayed using a $\mathrm{NADP}^{+} / \mathrm{NADPH}$ Quantification Colorimetric Kit (Biovision, Milpitas, CA), according to the manufacturer's instructions, and normalized to the control.

Other Methods. For details on measurement of intracellular ROS, chemicals, overexpression of $\mathrm{ROR} \alpha$ and knockdown of ROR $\alpha$ and PDK2, ATP measurement, measurement of luciferase activity, animal experiments, and immunohistochemical (IHC) staining and scoring, see the Materials and Methods section of the Supporting Information.

Ethics Statement. This study was approved by the institutional review board (IRB) of Kyunpook National University Hospital (IRB no.: KNUMCBIO_12-1007) and Keimyung University Dongsan Hospital (IRB no.: 11-256).

Statistical Analyses. Statistical analyses were performed using the Student $t$ test. Data are presented as the mean \pm standard error of the mean (SEM). Clinical data management and statistical analyses were performed using SPSS software (version 18.0; SPSS, Inc., Chicago, IL). The association between protein expression was evaluated using Pearson's $\chi 2$ test; $P<0.05$ was considered significant.

\section{Results}

Glutamine Is Required for Aerobic Glycolysis in Hepatoma Cells. We first determined the levels of glutaminase (GLS) 1, lactate dehydrogenase A (LDHA), and $\mathrm{PDH}$ phosphorylation as representative molecules crucial for glutamine and glucose metabolism in six human HCC tumor tissues and compared them with matched adjacent normal liver tissue. There was an increase in the levels of GLS1 protein, LDHA protein, and $\mathrm{PDH}$ phosphorylation in tumor tissue (Fig. 1A), which suggested a marked increase in glutamine catabolism and aerobic glycolysis, required for maintaining the TCA cycle and for biomass synthesis, respectively. By contrast, the $\mathrm{ROR} \alpha$ level in tumor tissue was markedly reduced, compared to the normal counterpart (Fig. 1A). Based on these data, we hypothesized that down-regulation of ROR $\alpha$ in hepatoma cells may contribute to altered glucose and glutamine metabolism, enabling cancer cell growth.

To better understand the role of ROR $\alpha$ in the interconnected glutamine and glucose metabolic pathways in hepatoma cells, including human $\mathrm{HB}$ cell line HepG2 and HCC cell line Hep3B, we investigated the effect of glutamine deprivation on aerobic glycolysis. Glucose uptake by HepG2 cells and Hep3B cells during glutamine deprivation was significantly reduced, compared to the control, and this reduction in glucose uptake was reversed after glutamine resupplementation (Fig. 1B). Next, we examined changes in the M2 isoform of pyruvate kinase (PKM2), which produces pyruvate from phosphoenolpyruvate (PEP), and in levels of LDHA, which metabolizes pyruvate to lactate. The results showed that glutamine deprivation attenuated the levels of phosphorylated PKM2 and of LDHA, and glutamine resupplementation caused the recovery of both, indicating that, under glutamine deprivation conditions, the conversion of PEP to pyruvate is increased, but the conversion of pyruvate to lactate is decreased (Fig. 1C). Because of this finding, we investigated whether glutamine starvation attenuated the expression of genes involved in biosynthetic pathways that are required for cancer cell proliferation. Depriving hepatoma cells of glutamine down-regulated the messenger RNA (mRNA) expression levels of 
A
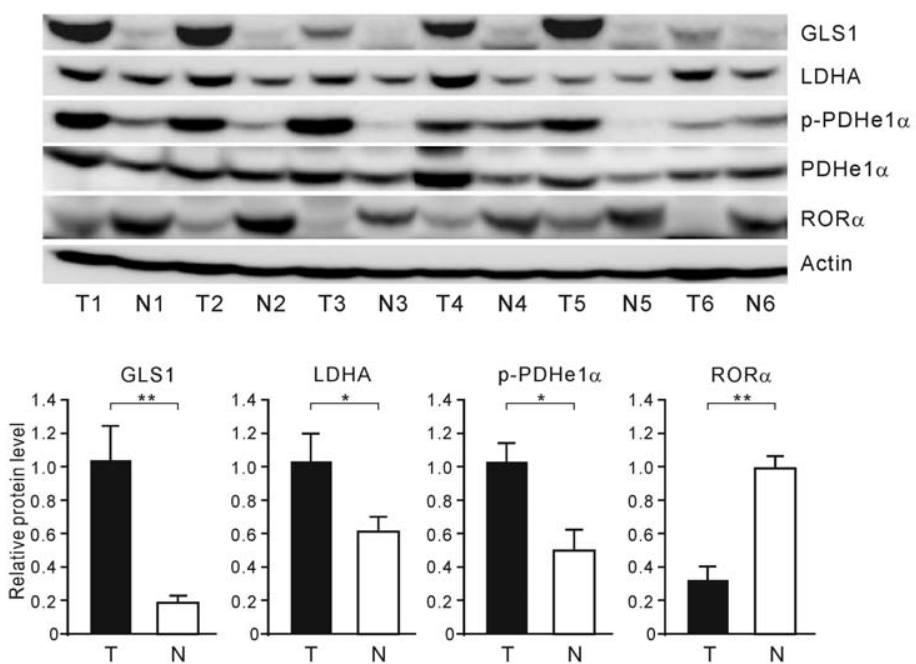

D
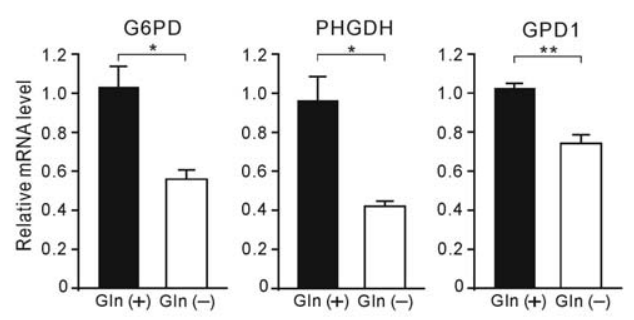

B

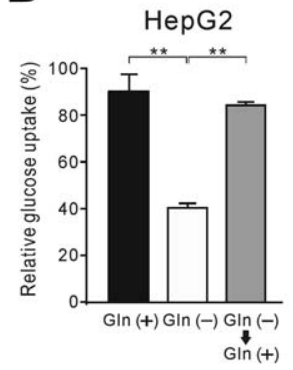

C

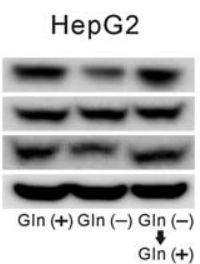

F

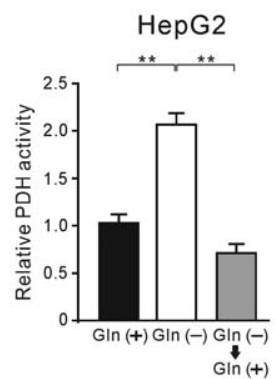

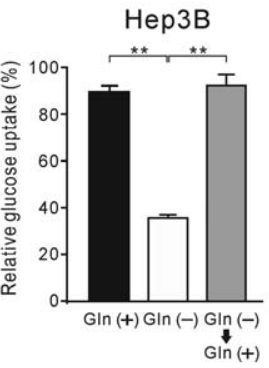

Hep3B

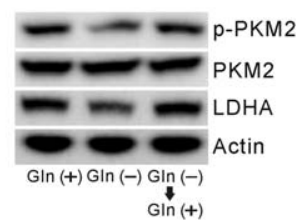

Hep3B

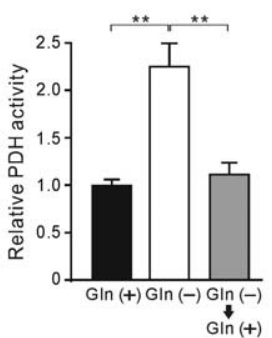

Fig. 1. Glutamine is required for aerobic glycolysis in hepatoma cells. (A) Protein levels of GLS1, LDHA, and ROR $\alpha$ and phosphorylation of PDHe1 $\alpha$ in human HCC. Human HCC tissue (T) and corresponding adjacent nontumor liver tissue (N) from 6 patients was analyzed by western blotting. Relative quantification of proteins by western blotting analysis. GLS1, LDHA, and ROR $\alpha$ were normalized relative to the level of actin, and $\mathrm{p}$-PDHe1 $\alpha$ was normalized relative to the level of PDHe1 $\alpha$. The level of the indicated protein in nontumor tissues was normalized with the level in the tumor tissues and is shown as a bar chart of the ratio. (B) Glucose uptake in hepatoma cells cultured in complete medium (including $10 \mathrm{mM}$ of glucose and $2 \mathrm{mM}$ of glutamine) or glutamine-free medium for 48 hours, or glutamine-free medium for 48 hours followed by complete medium for 24 hours. (C) Western blotting analysis of phosphorylated PKM2 and LDHA protein levels in hepatoma cells treated as in (B). (D) qRT-PCR analysis of relative expression of G6PD, PHGDH, and GPD1 mRNA in HepG2 cells cultured in the presence or absence of glutamine for 48 hours. (E) Western blotting analysis of PDK isoform protein levels and phosphorylation of PHDe1 $\alpha$ in HepG2 cells treated as in (B). (F) Relative PDH activity in hepatoma cells treated as in (B). Results represent the means ( \pm SEM) of three independent experiments performed in triplicate. Error bars represent the SEM. ${ }^{*} P<0.05 ;{ }^{*} P<0.01$. Abbreviations: Gln $(+)$, in the presence of glutamine; Gln $(-)$, in the absence of glutamine.

glucose-6-phosphate dehydrogenase (G6PD; of the hexosamine pathway), phosphoglycerate dehydrogenase (PHGDH; of the serine synthesis pathway), and glycerol-3-phosphate dehydrogenase 1 (GPD1; of the glycerol-3-phosphate synthesis pathway; Fig. 1D and Supporting Fig. 1A).

Pyruvate dehydrogenase kinase (PDK) is a critical regulator of the balance between pyruvate oxidation and lactate production. PDKs phosphorylate the gatekeeping mitochondrial enzyme PDHe $1 \alpha$ complex and inhibit its activity. Therefore, we tested whether glutamine deprivation or inhibition of glutaminolysis influenced PDK expression in hepatoma cells. The results showed that the abundance and activity of PDK1-3 proteins were reduced by glutamine deprivation (Fig.
$1 \mathrm{E}$ and Supporting Fig. 1B). Conversely, resupplementation with glutamine reversed the reduction in the levels of PDK1-3 and phospho-PDHe1 $\alpha$ (Fig. 1E), and recovered $\mathrm{PDH}$ activity (Fig. $1 \mathrm{~F}$ ). The relative quantities of PDK3 and 4 transcripts in HepG2 cells were very low, compared to those of PDK1 and 2 (Supporting Fig. 1C), suggesting that PDK1 and 2 have major roles in the modulation of $\mathrm{PDHe} 1 \alpha$ activity in the absence of glutamine. To examine whether chemical inhibitors of glutamine metabolism induce similar changes, we treated HepG2 cells with the glutaminase inhibitor, bis-2-(5-phenylacetamido-1,2,4thiadiazol-2-yl)ethyl sulfide (BPTES), or with aminooxyacetate (AOA), a chemical inhibitor of glutamatedependent transaminases. Consistent with the results 
after glutamine deprivation, PDK1-3 levels and phosphorylation of PDHe1 $\alpha$ were attenuated by treatment with these inhibitors (Supporting Fig. 1D). These results indicate that glutamine deprivation in hepatoma cells promotes a shift of glucose metabolism toward oxidative phosphorylation (OXPHOS), rather than aerobic glycolysis or biosynthesis.

RORa Expression Is Induced in Hepatoma Cells Upon Glutamine Deprivation. Previous studies have demonstrated that ROR $\alpha$ is crucial for the stabilization of $\mathrm{p} 53,{ }^{18}$ which is known to reduce PDK2 protein levels. ${ }^{20}$ Thus, we speculated that $\mathrm{ROR} \alpha$ controls altered glucose metabolism by up-regulation of $\mathrm{p} 53$. To explore this possibility, we assessed whether ROR $\alpha$ expression is up-regulated by glutamine deprivation. Indeed, glutamine deprivation of hepatoma cells led to an increase in the expression of $\operatorname{ROR} \alpha$, which was returned to basal levels by resupplementation with glutamine (Fig. 2A). We also found that chemical inhibitors of glutaminolysis (BPTES and AOA) increased the abundance of $\operatorname{ROR} \alpha$ (Supporting Fig. 2A,B). Glutamine supports redox homeostasis through NADPHdependent GSH and thioredoxin systems; thus, we examined whether cellular ROS levels are increased by glutamine deprivation and whether such an increase leads to induction of $\operatorname{ROR} \alpha$ expression. Glutamine depletion increased the level of cellular ROS and the $\mathrm{NADP}^{+} / \mathrm{NADPH}$ ratio in hepatoma cells, which was consistent with a reduced level of GSH/GSSG ratio (Fig. 2B-D). We also found that the level of thioredoxin-interacting protein (TXNIP), an endogenous thioredoxin inhibitor, was increased in the absence of glutamine (Fig. 2E). Moreover, antioxidants $\mathrm{N}$-acetylcysteine and GSH prevented glutamine depletion-induced ROR $\alpha$ expression (Fig. 2F).

Overexpression of ROR Alters Glucose Utilization in Hepatoma Cells by Down-Regulation of PDK2. We next investigated whether $\operatorname{ROR} \alpha$ activation mimics glutamine deprivation-induced alteration of glucose metabolism. Adenovirus (Ad)-mediated overexpression of $R O R \alpha$ in hepatoma cells decreased glucose uptake (Fig. 3A and Supporting Fig. 3A), and the expression of G6PD, PHGDH, and GPD1 was also attenuated upon $\mathrm{ROR} \alpha$ overexpression (Fig. 3B and Supporting Fig. 3B). Furthermore, ROR $\alpha$ overexpression decreased the abundance of PDK2 protein and phosphorylation of $\mathrm{PDHe} 1 \alpha$ in a dose-dependent manner (Fig. 3C) and subsequently increased $\mathrm{PDH}$ activity (Fig. 3D). However, ROR $\alpha$ overexpression had little effect on PDK1, 3, and 4. Next, using small interfering RNA (siRNA) knockdown, we tested whether the inhibition of PDK2 expression upon glu- tamine deprivation was ROR $\alpha$ dependent. Suppression of PDK2 and phospho-PDHe1 $\alpha$ in HepG2 cells upon glutamine deprivation was markedly reversed by inhibition of endogenous $\mathrm{ROR} \alpha$ induction (Fig. 3E). The effects of ROR $\alpha$ on glucose metabolism in HepG2 cells were further examined with a chemical activator and a chemical inhibitor of $\operatorname{ROR} \alpha$. In concordance with our previous data, we found that the $\operatorname{ROR} \alpha$ activator, SR1078, decreased glucose uptake and attenuated PDK2 protein levels (Supporting Fig. 3C,D). By contrast, the ROR $\alpha$ inhibitor, SR3335, significantly recovered PDK2 protein levels and PDHe1 $\alpha$ phosphorylation (Supporting Fig. 3E).

Cancer cells produce ATP from both aerobic glycolysis and OXPHOS; thus, we investigated the relative contribution of aerobic glycolysis and OXPHOS in the production of ATP with regard to $R O R \alpha$. To ascertain the proportion of ATP generated from OXPHOS versus glycolysis, we limited OXPHOS ATP synthesis by treatment with oligomycin, an inhibitor of ATP synthase, in hepatoma cells overexpressing $\operatorname{ROR} \alpha$ (Ad-ROR $\alpha$ treated). We found that in Admock treated hepatoma cells, OXPHOS contributed only marginally to ATP synthesis, with a higher proportion of the intracellular ATP content being provided by glycolysis, with a marked increase in glycolytic ATP. By contrast, in Ad-ROR $\alpha$-treated hepatoma cells, a larger proportion of ATP came from OXPHOS (Fig. 3F and Supporting Fig. 3F), indicating that $R O R \alpha$ increases the contribution of ATP from OXPHOS, rather than from glycolysis in hepatoma cells.

Inbibition of PDK2 Expression by ROR Is p21

Dependent. To explore the molecular mechanisms responsible for PDK2 down-regulation, we investigated the link between expression of PDK2 and ROR $\alpha$. It has been reported that $\mathrm{p} 53$ and $\mathrm{p} 21$ are ROR target genes. ${ }^{18,21}$ In accord with previous studies, overexpression of ROR $\alpha$ or treatment with SR1078 in HepG2 cells markedly increased p53 and p21 protein levels, but decreased phosphorylated retinoblastoma protein (pRb) levels (Fig. 4A,B). Because ROR $\alpha$ led to downregulation of PDK2 in p53-null Hep3B cells (Fig. 3C, right panel), we further examined whether $\operatorname{ROR} \alpha$ regulates $\mathrm{p} 21$ in a p53-independent manner. The results showed that overexpression of $\operatorname{ROR} \alpha$ up-regulates $\mathrm{p} 21$ protein level in Hep3B cells, indicative of $\mathrm{p} 53$ indepndent function of $\operatorname{ROR} \alpha$ (Supporting Fig. 4A). Moreover, overexpression of ROR $\alpha$ in another human HCC cell line, Huh7, showed increased expression of p21 and attenuation of PDK2 expression without alteration of p53 protein levels (Fig. 4C), 


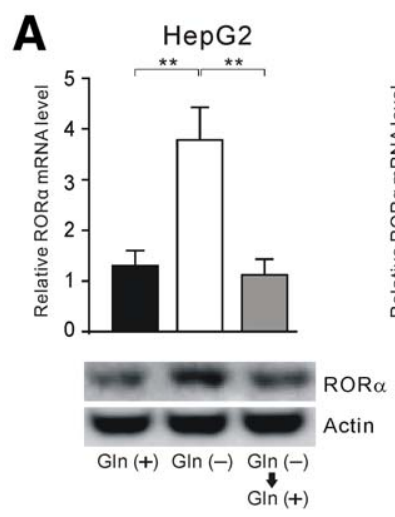

D

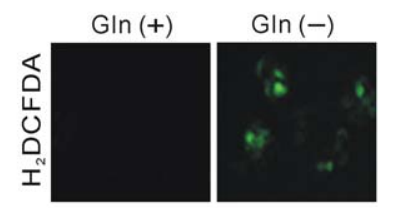

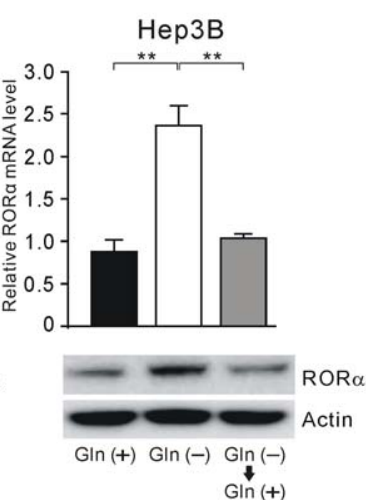

E

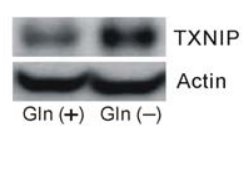

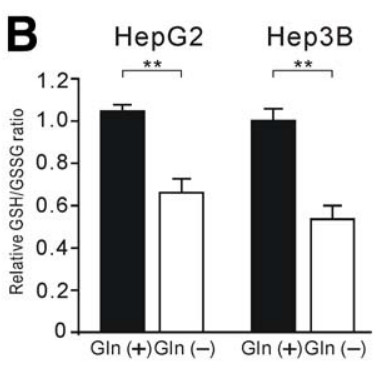
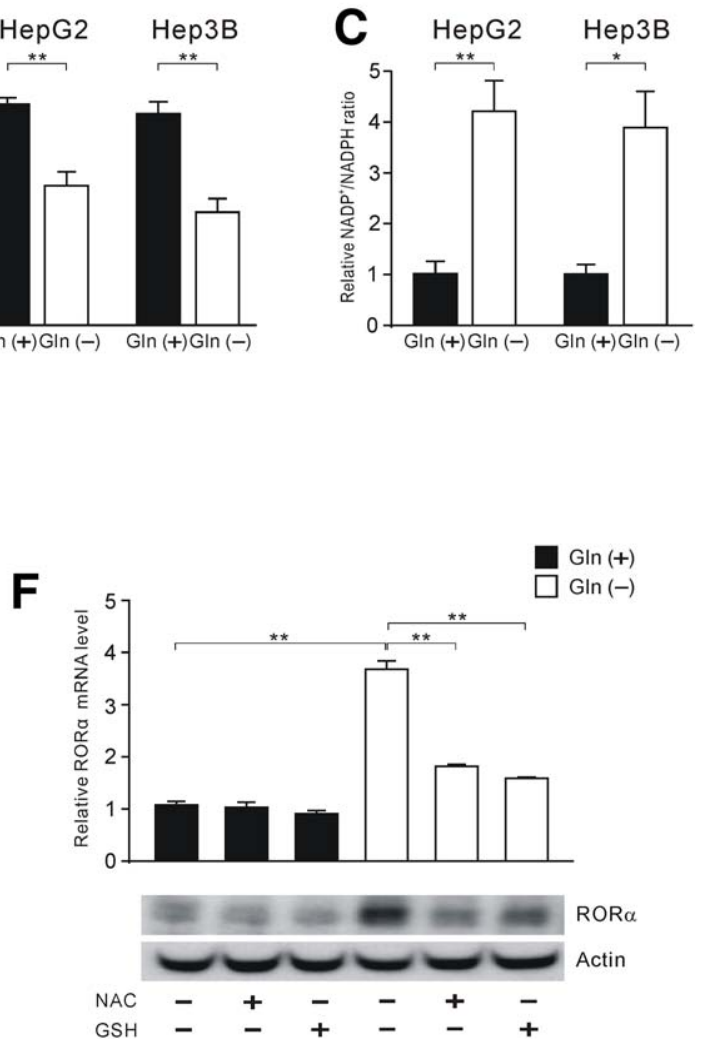

Fig. 2. ROR $\alpha$ expression is induced after glutamine deprivation through ROS production. (A) qRT-PCR of ROR $\alpha$ mRNA and protein levels by western blotting analysis in hepatoma cells cultured in complete medium (including $10 \mathrm{mM}$ of glucose and $2 \mathrm{mM}$ of glutamine) or glutaminefree medium for 24 hours, or glutamine-free medium for 24 hours followed by complete medium for 24 hours. (B) Measurement of the GSH/ GSSH ratio and (C) $\mathrm{NADP}^{+} / \mathrm{NADPH}$ ratio in hepatoma cells in the presence or absence of glutamine for 24 hours. Results were normalized to those obtained in complete medium. (D) 2',7'-dichlorodihydrofluorescein diacetate (DCF-DA) staining, as a measure of ROS, before and after glutamine deprivation. HepG2 cells were glutamine starved for 24 hours and stained with DCF-DA. DCF fluorescence was visualized by fluorescence microscopy. (E) Western blotting analysis of TXNIP protein levels in HepG2 cells in the presence or absence of glutamine for 24 hours. (F) qRTPCR of ROR $\alpha$ mRNA and protein levels by western blotting analysis in HepG2 cells. Cells were cultured in complete or glutamine-free medium with or without treatment with NAC $(8 \mathrm{mM})$ or GSH $(8 \mathrm{mM})$ for 24 hours. Error bar represents the SEM. ${ }^{*} P<0.05 ;{ }^{*} * P<0.01$. Abbreviations: Gln $(+)$, in the presence of glutamine; Gln $(-)$, in the absence of glutamine. NAC, N-acetylcysteine.

demonstrating that $\mathrm{ROR} \alpha$ regulates $\mathrm{p} 21$ and PDK2 protein levels in hepatoma cells regardless of p53 expression. Interestingly, $\mathrm{ROR} \alpha$ overexpression in the transformed human hepatocyte cell line, THLE-2, did not change p21, PDK2, or phospho-PDHe1 $\alpha$ levels (Supporting Fig. 4B), suggesting that the role of $\mathrm{ROR} \alpha$ in the reprogramming of glucose metabolism is limited to hepatoma cells.

After confirming the p53-independent effect of ROR $\alpha$ on the level of PDK2, we then examined whether effect of ROR $\alpha$ on the level of PDK2 is p21 dependent. Upon reduction of p21 by siRNA in HepG2 and Huh7 cells, the effect of ROR $\alpha$ on the PDK2 protein level was abolished (Fig. 4D). Given that p21 inhibits E2F transcriptional activity, and E2F increases PDK2 transcription, ${ }^{20}$ it was necessary to examine whether $\mathrm{ROR} \alpha$ decreased E2F activity. Overexpression of $\operatorname{ROR} \alpha$ attenuated the activity of
E2F on the PDK2 promoter (Fig. 4E). We further examined whether down-regulation of PDK2 affects p21 protein levels and E2F activity in p53-positive or -negative cells. Indeed, we found that short hairpin (sh)-PDK2 increased p21 protein levels and decreased $[\mathrm{E} 2 \mathrm{~F}] \times 4$ luciferase reporter, which contains four E2F-binding sites, ${ }^{22}$ in both HeG2 and Hep3B cells (Supporting Fig. 5A,B), suggesting that interaction of PDK2 with p21- and E2F-binding activity are p53 independent. Collectively, these results indicate that ROR $\alpha$-induced down-regulation of PDK2 levels is resulted from inhibition of E2F activity through upregulation of $\mathrm{p} 21$.

ROR Activation Inbibits Hepatoma Cell Growth. Glutamine is an essential nutrient for proliferating cancer cells, and HCCs exhibit a high rate of glutamine consumption. ${ }^{23}$ Thus, it is expected that blocking glutamine catabolism in hepatoma cells might 

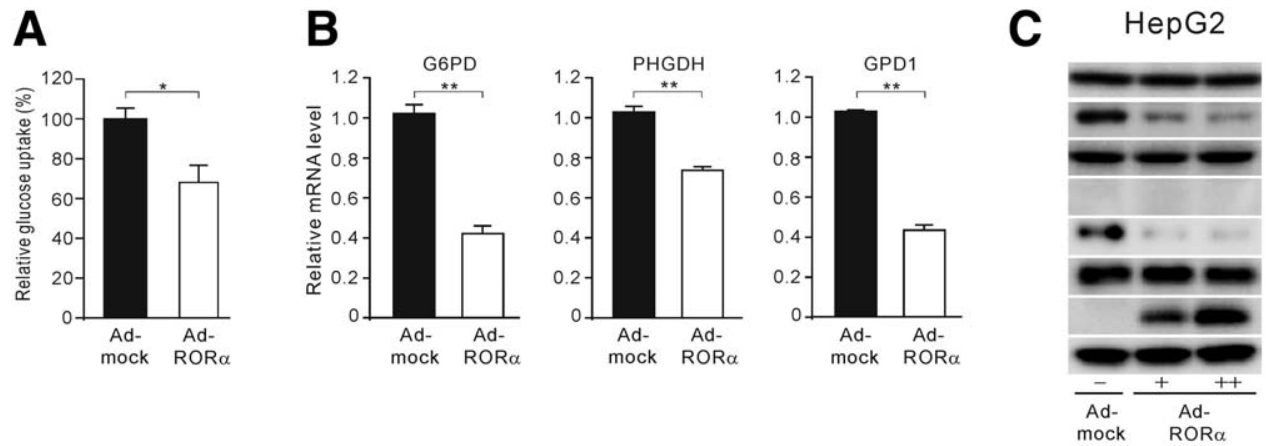

Hep3B

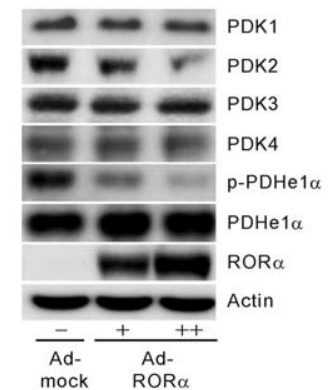

D
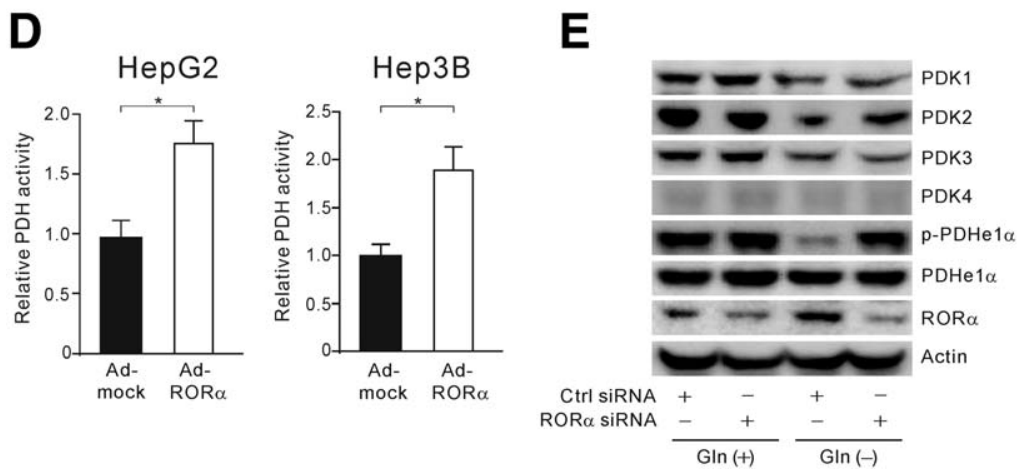

$\mathbf{F}$

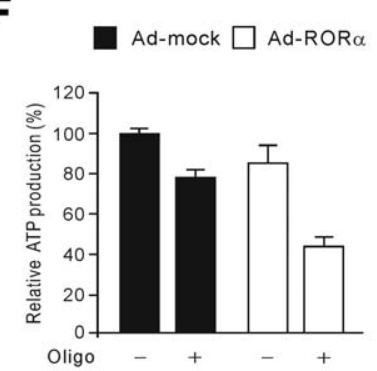

Fig. 3. Overexpression of ROR $\alpha$ alters glucose utilization in hepatoma cells by down-regulation of PDK2. (A) Glucose uptake in HepG2 cells with Ad-mediated overexpression of ROR $\alpha$ (100 multiplicities of infection [MOI]). Bar charts represent fold change of relative glucose uptake in Ad-ROR $\alpha$ HepG2 cells after normalization to that of Ad-mock cells. (B) qRT-PCR analysis of relative mRNA expression of G6PD, PHGDH, and GPD1 in HepG2 cells overexpressing ROR $\alpha(100 \mathrm{MOI})$. (C) Western blotting analysis of PDK isoform proteins and PHDe $1 \alpha$ phosphorylation levels in HepG2- and Hep3B-overexpressing ROR $\alpha$ cells (+, $50 \mathrm{MOI}$; ++, $100 \mathrm{MOI}$ ). (D) Relative PDH activity in HepG2- and Hep3B-overexpressing ROR $\alpha$ cells $(100 \mathrm{MOI})$. (E) Western blotting analysis of the effect of siRNA-ROR $\alpha$ on PDK isoform proteins and PHDe $1 \alpha$ phosphorylation levels in HepG2 cells in the presence or absence of glutamine for 48 hours. (F) Relative ATP levels in control or HepG2 cells overexpressing ROR $\alpha$ (100 $\mathrm{MOI})$ with or without treatment with oligomycin $(1 \mu \mathrm{M})$. Error bar represents the SEM. ${ }^{*} P<0.05 ; * * P<0.01$. Abbreviations: Gln $(+)$, in the presence of glutamine; Gln $(-)$, in the absence of glutamine.

result in decreased proliferation. Glutamine depletion or treatment with chemical inhibitors of glutamine metabolism (BPTES or AOA) caused a significant decrease in the number of proliferating hepatoma cells in a time- or dose-dependent manner, respectively (Fig. 5A,B). Cell proliferation was also significantly inhibited by $\operatorname{ROR} \alpha$ overexpression or SR1078 treatment in both HepG2 and Hep3B cells (Fig. 5C,D). Moreover, we found that inhibition of HepG2 cell proliferation under glutamine-free conditions was partially rescued by the addition of the $R O R \alpha$ inverse agonist, SR3335 (Fig. 5E), indicating that $\operatorname{ROR} \alpha$, at least in part, mediates glutamine depletion-induced inhibition of cell proliferation.

ROR a Activation Inhibits Tumor Growth. To investigate whether modulation of ROR $\alpha$ and PDK2 could affect tumor growth in vivo, we injected Hep3B cells overexpressing $\operatorname{ROR} \alpha$ subcutaneously (SC) into nude mice. Tumors in mice injected with Hep3B cells overexpressing $\operatorname{ROR} \alpha$ were significantly smaller than those of control mice (mean tumor volume: 319.25 vs. $\left.40.75 \mathrm{~mm}^{3} ; P<0.05\right)$ and exhibited higher induction of p21 and lower induction of PDK2 and phosphoPDHe1 $\alpha$ than control mice (Fig. 6A,B). Furthermore, mice injected with PDK2-knocked down Hep3B cells developed remarkably smaller tumors than those of control mice (mean tumor volume: 1159.67 vs. $388.33 \mathrm{~mm}^{3}$; $P<0.05$; Fig. 6C). Next, to examine the effect of SR1078 on proliferation of established tumors, we injected HepG2 cells SC into nude mice. Once the tumors were palpable, mice were injected daily intraperitoneally with either SR1078 or dimethyl sulfoxide (DMSO). At day 12 postinoculation, tumors in mice treated with SR1078 were significantly smaller than those of controls (mean tumor volume: 226.43 vs. $73.50 \mathrm{~mm}^{3} ; P<0.01$; Fig. $\left.6 \mathrm{D}, \mathrm{E}\right)$. Xenografted tumor tissue in mice treated with SR1078 had reduced levels of PDK2 and PDH phosphorylation, but increased p21 levels (Fig. 6F), compared to tumor tissue from control mice. Altogether, these data corroborate the effects of ROR $\alpha$ on p21 and PDK2 observed in vitro. 
A

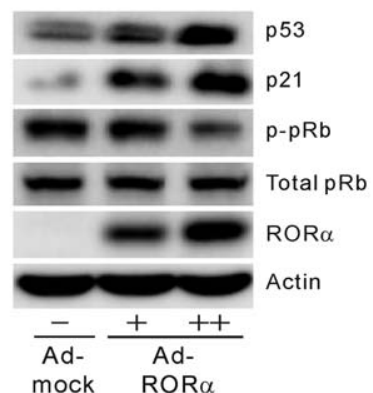

B

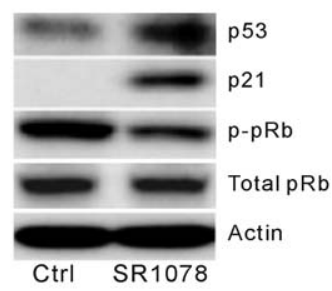

C

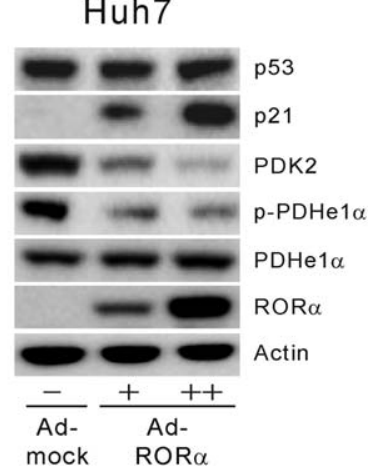

D

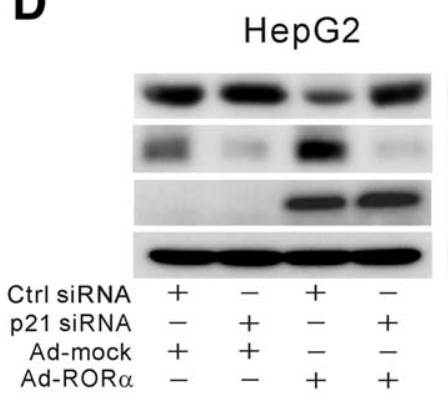

Huh7

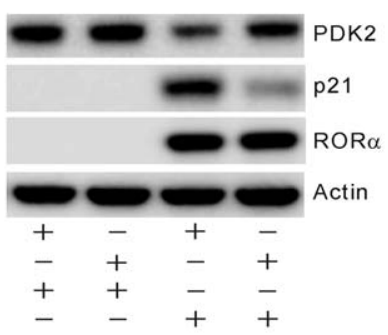

E

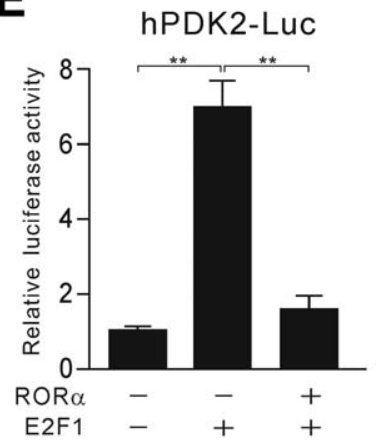

Fig. 4. Inhibition of PDK2 expression by ROR $\alpha$ is p21-dependent. (A) Western blotting analysis of p53, p21, and p-pRb protein levels, in HepG2 cells overexpressing ROR $\alpha$ (,+ 50 multiplicities of infection [MOI];,$++ 100 \mathrm{MOI})$. (B) Effect of SR1078 (10 $\mu \mathrm{M})$ on p53, p21, and p-pRb expression in HepG2 cells determined by western blotting analysis. (C) Western blotting analysis of p21, PDK2, and phospho-PDHe1 $\alpha$ protein levels in Huh7 overexpressing ROR $\alpha(+, 50 \mathrm{MOI}$;,$++ 100 \mathrm{MOI})$. (D) Western blotting analysis of the effect of siRNA-p21 on ROR $\alpha$-induced reduction of PDK2 protein levels in HepG2 and Huh7 cells. (E) Effect of ROR $\alpha$ on E2F1-stimulated human PDK2 promoter activity. Cells were cotransfected with the human PDK2 promoter and E2F expression vector. Luciferase activity was assayed and normalized to a transfection control. Error bar represents the SEM. ${ }^{*} P<0.05$; ${ }^{* *} P<0.01$.

Less ROR $\alpha$ Is Expressed in Tumor Tissue of 187 Patients With HCC. Finally, we analyzed the expression of $\mathrm{ROR} \alpha$ in tumors from a cohort of HCC patients after liver resection and compared its expression with adjacent nontumor tissue obtained from the same patients. The basal characteristics of patients are presented in Table 1. IHC analysis of a panel of 187 pairs of HCC and adjacent nontumor tissue samples revealed that whereas 144 cases $(77.0 \%)$ of nontumor samples were $\operatorname{ROR} \alpha$ positive, only 52 cases $(28.9 \%)$ of tumor lesions were positive (Fig. 7A,B, left panel). Although not statistically significant, patients with loss of tumor $\operatorname{ROR} \alpha$ expression tended to have tumor node metastasis (TNM) stage III disease $(P=0.071$; Fig. $7 \mathrm{~B}$, right panel). To correlate the expression of p21 and PDK2 with ROR $\alpha$ in tumor tissues, we measured the immunoreactivity of p21 and PDK2 and compared it with $\operatorname{ROR} \alpha$ expression. Importantly, $\operatorname{ROR} \alpha$-positive tumor tissues were prone to have higher p21 immunoreactivity (negative, 18.5\%; 1+, $79.6 \% ; 2+, 1.9 \%$ vs. negative, $88.6 \% ; 1+, 11.4 \%$; $2+, 0 \% ; P<0.001$ ) and lower PDK2 activity (nega- tive, $7.4 \% ; 1+, 63 \% ; 2+, 25.9 \% ; 3+, 3.7 \%$ vs. negative, $3.8 \% ; 1+, 36.4 \% ; 2+, 52.3 \% ; 3+, 7.6 \%$; $P=0.003$ ) than $\mathrm{ROR} \alpha$-negative tumor tissues (Fig. 7C). Statistical analysis showed that ROR $\alpha$-positive tumor tissues had a strong positive association with p21 expression and an inverse negative association with PDK2 activity (Fig. 7D; $P<0.001$ and $P=0.003$, respectively). However, we found no statistical significance for the association between $\operatorname{ROR} \alpha$ expression and p53 expression (data not shown).

\section{Discussion}

The present study demonstrates that $\mathrm{ROR} \alpha$ reprograms glucose metabolism in glutamine-deficient hepatoma cells. Inhibiting the supply of glutamine markedly increased the expression of $\mathrm{ROR} \alpha$ in hepatoma cells, and then $\operatorname{ROR} \alpha$ reduced the expression of PDK2 and shifted pyruvate toward oxidative phosphorylation, rather than glycolysis and biosynthesis (Fig. 8). Furthermore, we found that $\mathrm{ROR} \alpha$ inhibited hepatoma growth both in vitro and in a xenograft model in vivo. 

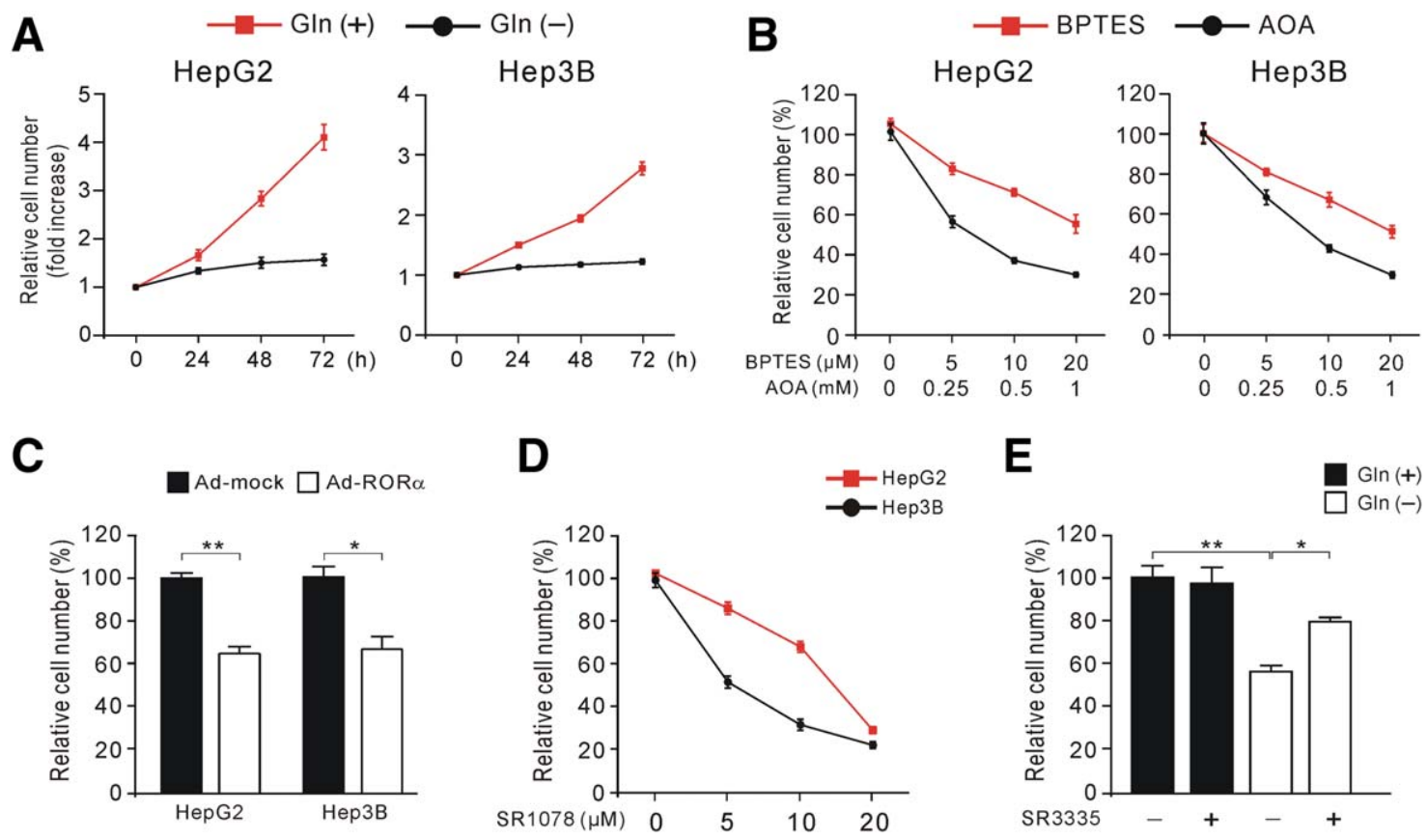

Fig. 5. ROR $\alpha$ activation inhibits hepatoma cell growth. (A) Growth curve analysis of hepatoma cells. Cells were cultured in the presence or absence of glutamine. (B) Growth curve analysis of hepatoma cells treated with the indicated doses of BPTES or AOA for 48 hours. (C) Growth of hepatoma cells with Ad-mediated overexpression of control vector or ROR $\alpha$ (100 multiplicities of infection). (D) Growth curve analysis of hepatoma cells treated with the indicated doses of SR1078 for 48 hours. (E) Growth of HepG2 cells in the presence or absence of glutamine for 48 hours after pretreatment with SR3335 $(20 \mu \mathrm{M})$ for 6 hours. Error bar represents the SEM. ${ }^{*} P<0.05$; ${ }^{* *} P<0.01$. Abbreviations: Gln $(+)$, in the presence of glutamine; Gln $(-)$, in the absence of glutamine.

A

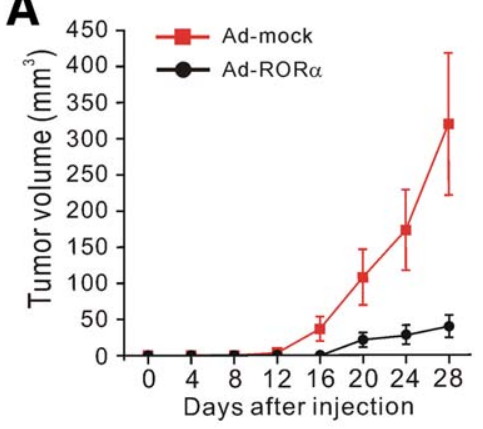

D

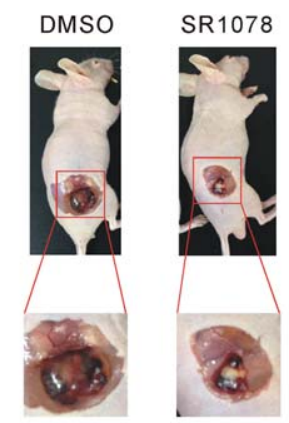

B

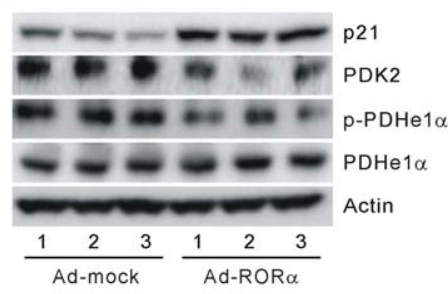

$\mathbf{E}$

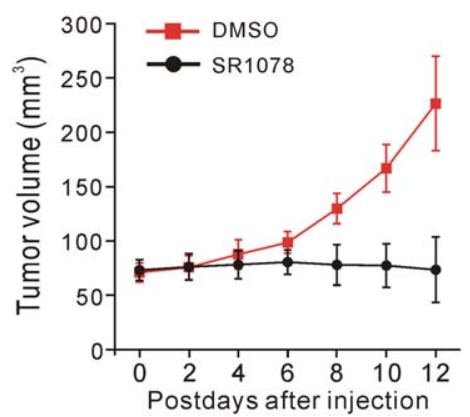

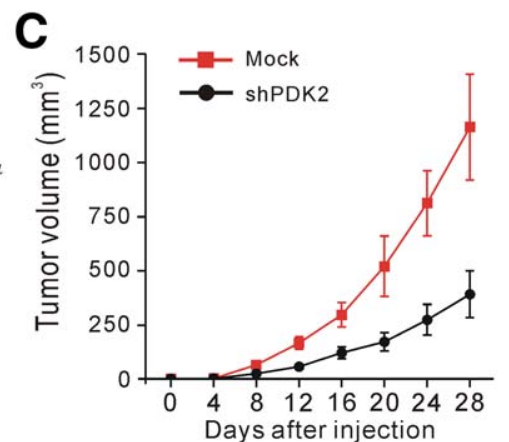

$\mathbf{F}$

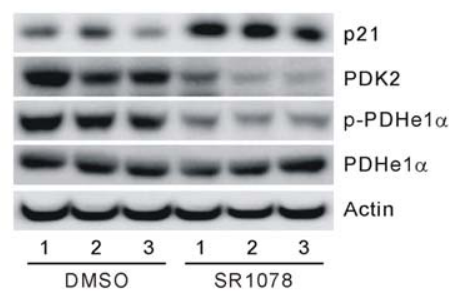

Fig. 6. ROR $\alpha$ activation inhibits tumor growth. (A) Representative growth curve of tumors resulting from xenografted Hep3B cells overexpressing ROR $\alpha$ in nude mice. (B) Effect of overexpression of ROR $\alpha$ on p21, PDK2, and phospho-PDHe1 $\alpha$ protein levels in tumor tissue from xenografted mice, determined by western blotting analysis. (C) Representative growth curve of tumors resulting from xenografted Hep3B cells with PDK2 knockdown in nude mice. (D) Representative image and (E) growth curve of tumors resulting from xenografted HepG2 cells in nude mice treated with or without SR1078. (F) Effect of SR1078 on p21 and PDK2 protein levels and phosphorylation of PHDe1 $\alpha$ in tumor tissue from xenografted mice, determined by western blotting analysis. Error bar represents the SEM. 
Table 1. Clinical Characteristics of Patients With Resected HCC

\begin{tabular}{lc}
\hline Variables & No. of Patients \\
\hline All cases & 187 \\
Gender (male/female) & $156 / 31$ \\
Mean age & $55.68 \pm 10.38$ \\
HCC cause & \\
$\quad$ Alcohol & 14 \\
HBV & 134 \\
HCV & 14 \\
HBV+HCV & 5 \\
Other causes & 20 \\
TNM stage & \\
I-II & 109 \\
III & 78 \\
\hline
\end{tabular}

$\mathrm{HBV}$, hepatitis B virus; $\mathrm{HCV}$, hepatitis C virus.

Cancer cells exhibit a metabolic phenotype different from that of normal cells; they show increased aerobic glycolysis and rely on glutamine to support their growth and proliferation. ${ }^{24}$ Accordingly, the metabolism of glutamine and glucose is recognized as a promising therapeutic target for the cancer treatment. However, little is known about the metabolic relationship between glucose and glutamine in liver cancer. In

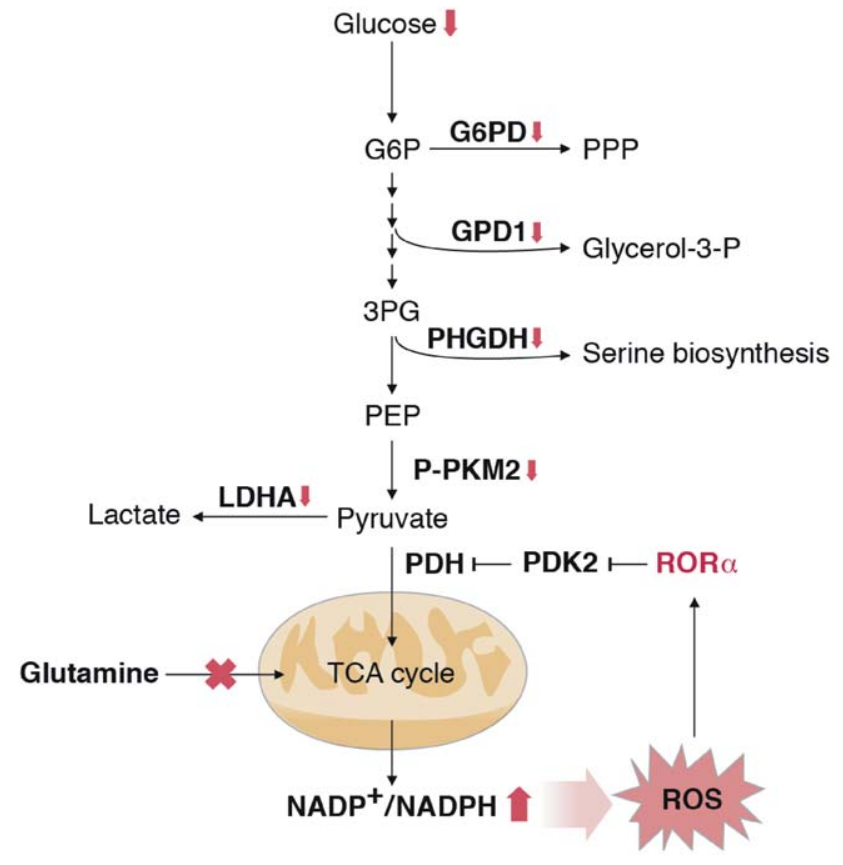

Fig. 8. Schematic drawing of ROS-induced activation of ROR $\alpha$ decreasing PDK2 and shifting pyruvate to oxidative phosphorylation, rather than toward glycolysis and biosynthesis. Abbreviations: 3PG, 3phospho-D-glycerate; PPP, pentose phosphate pathway.
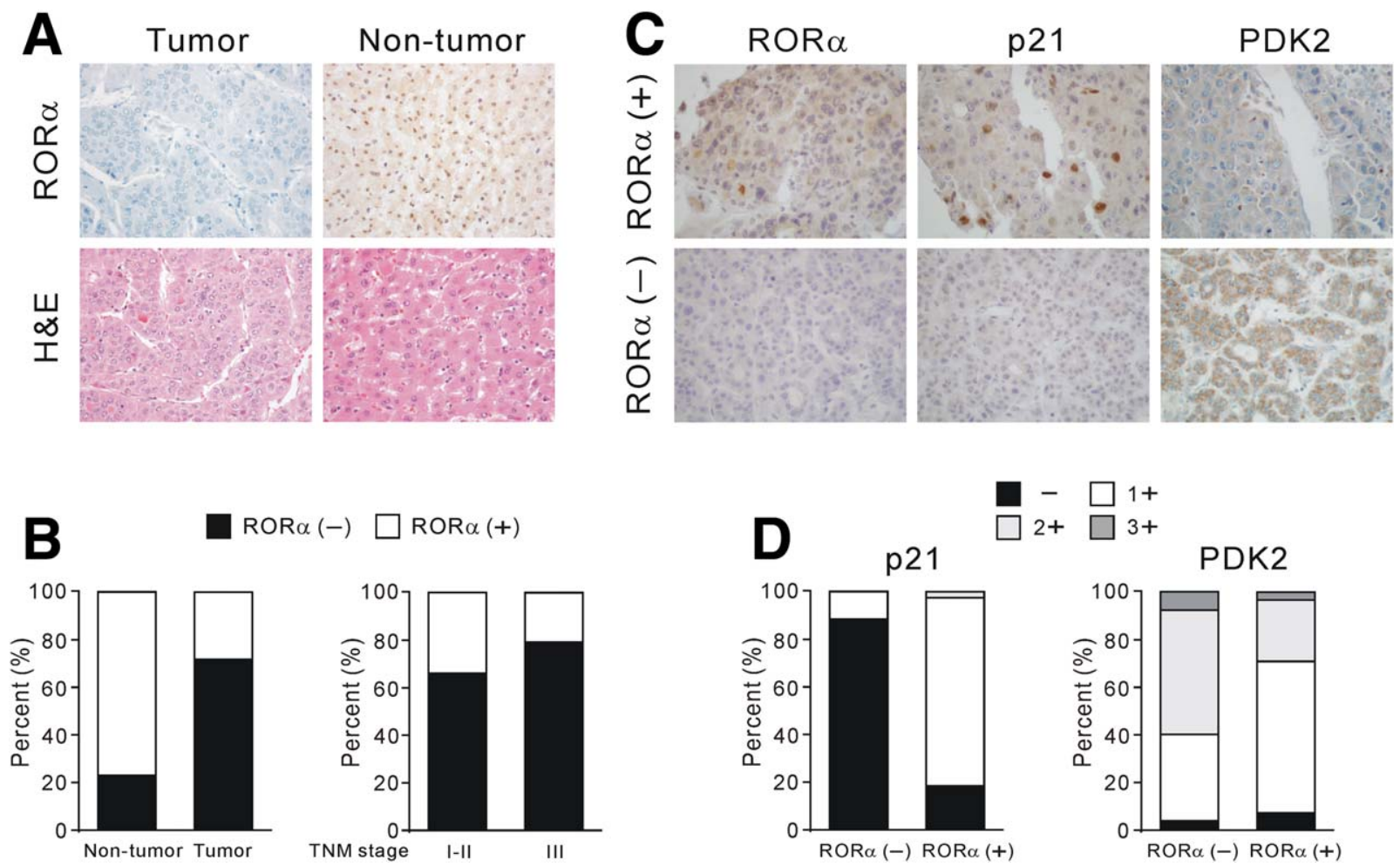

Fig. 7. ROR $\alpha$, p21, and PDK2 expression in tumor tissue of 187 patients with HCC. (A) Data from 187 surgically resected human HCC tumor and corresponding adjacent nontumor tissue. Representative IHC analyses of ROR $\alpha$ expression and H\&E staining in the tumor lesion and adjacent nontumor tissue (original magnification: $\times 200$ ). (B) Distribution of positive or negative ROR $\alpha$ staining by IHC in tumor and adjacent nontumor tissue (left panel). The association of ROR $\alpha$-positive staining with TNM stage (right panel). (C) Representative IHC analyses of p21 and PDK2 expression according to ROR $\alpha$ expression in the tumor lesion (original magnification: $\times 400$ ). (D) Correlation between expression of ROR $\alpha$ and expression of p21 and PDK2 in tumor lesion. Abbreviation: H\&E, hematoxylin-eosin. 
the present study, we found that glutamine depletion reduced glucose uptake, PKM2 phosphorylation, and expression of LDHA. Expression of genes involved in biosynthetic pathways, ${ }^{25}$ including G6PD, PHGDH, and GPD1, was also down-regulated upon glutamine deprivation. In addition, when glutamine was depleted, hepatoma cells showed decreased PDK abundance and PDH phosphorylation, both of which are critical regulators of pyruvate oxidation. Treatment with chemical inhibitors of glutamine metabolism (BPTES and AOA) caused a significant reduction in the number of proliferating hepatoma cells. These results indicate that glutamine deprivation inhibits the proliferation of hepatoma cells by shifting glucose metabolism toward OXPHOS, rather than aerobic glycolysis or biosynthesis.

Although therapies targeting glutamine metabolism effectively inhibit cancer cell proliferation, ${ }^{7}$ the efficacy and toxicity of pharmacological inhibitors of glutamine metabolism in cancer patients remain largely unknown. Therefore, it is necessary to develop a novel strategy that mimics the metabolic changes observed in glutamine-depleted tumor tissue without disrupting glutamine metabolism in normal tissue. Here, we also showed that glutamine deprivation markedly increased the expression of $\mathrm{ROR} \alpha$ in hepatoma cells. It is known that glutamine contributes to redox balance through the NADPH-dependent GSH and thioredoxin systems ${ }^{26}$; indeed, the present study showed that glutamine depletion increased the levels of cellular ROS and the $\mathrm{NADP}^{+} / \mathrm{NADPH}$ ratio, whereas it reduced the GSH/ GSSG ratio. Moreover, antioxidants prevented glutamine depletion-induced $\operatorname{ROR} \alpha$ expression, indicating that glutamine deprivation-induced ROS generation specifically promotes induction of $\operatorname{ROR} \alpha$. Interestingly, overexpression of ROR $\alpha$ in hepatoma cells altered glucose metabolism, which was observed during glutamine starvation; this phenomenon was manifested through attenuated glucose uptake, the expression of three enzymes essential for biosynthetic pathways, and enhanced PDH activity. However, although ROR $\alpha$ overexpression was associated with a reduction in PDK2 protein level, it had little effect on expression of PDK1 or PDK3, indicating that $\mathrm{ROR} \alpha$-induced reduction in PDK2 level could modulate PDH activity and mimic glutamine deprivation-induced reprogramming of glucose metabolism without affecting the expression of other PDKs. Moreover, we found that PDK2 knockdown with shRNA inhibited hepatoma growth, supporting the notion that ROR $\alpha$-induced PDK2 inhibition alone can induce the metabolic change that effectively suppresses hepatomoa cell growth. Indeed, we found that, in accord with inhibition of hepatoma growth by glutamine depri- vation, activation of $\mathrm{ROR} \alpha$ inhibited hepatoma proliferation. Additionally, we were able to confirm the antitumor effect of $\mathrm{ROR} \alpha$ activation in a xenograft model in vivo.

$\mathrm{ROR} \alpha$ has recently been identified as a positive regulator of p53 by increasing its transcriptional activity and protein stability. ${ }^{18}$ p53 is also known to activate $\mathrm{PDH}$ through p21-dependent transcriptional repression of PDK2. ${ }^{20}$ In the present study, we obtained similar results to those of previous studies showing that ROR $\alpha$ increased p53 and p21 protein levels. However, the results form p53-null Hep3B cells and HepG2 and Huh7 cells transfected with siRNA against p21 showed that ROR $\alpha$-mediated reduction in PDK2 levels resulted from up-regulation of $\mathrm{p} 21$, rather than $\mathrm{p} 53$. Collectively, these results indicate that $\mathrm{ROR} \alpha$ mediates the metabolic switch from aerobic glycolysis to OXPHOS by decreasing PDK2 levels through up-regulation of $\mathrm{p} 21$.

Importantly, this study showed that tumors from 187 patients with HCC expressed less $\mathrm{ROR} \alpha$ than adjacent nontumor tissue, suggesting that $\operatorname{ROR} \alpha$ activation could be a promising therapeutic target for HCC. Patients whose tumors lost $\mathrm{ROR} \alpha$ expression tended to have a higher tumor stage, although this was not statistically significant. Given that the study cohort consisted of patients who had undergone curative tumor resection, our study is limited to relatively early-stage HCC. Expression of $\operatorname{ROR} \alpha$ may be significantly associated with tumor stage; therefore, advanced-stage disease warrants future investigation. The clinical data support the in vitro and xenograft findings showing that $\mathrm{ROR} \alpha$ expression hampers glucose utilization and reduces HCC proliferation; thus, compounds that activate ROR $\alpha$ have potential for the treatment of HCC.

In conclusion, the data presented herein show that glutamine depletion in hepatoma cells promotes glucose oxidation to maintain the energy required for survival, but halts the biosynthetic pathways required for cell growth. ROR $\alpha$ mediates this reprogramming of glucose metabolism in hepatoma in response to glutamine deficiency. Given that a pharmacological activator of $\operatorname{ROR} \alpha$ could potentially act synergistically with inhibitors of glutamine metabolism in liver cancer, these findings have implications for future therapeutic approaches to liver cancer.

Acknowledgment: The biospecimens for this study were provided by the Kyungpook National University Hospital Biobank and Keimyung Human Bio Resource Bank (KHBB), a member of the National Biobank of Korea, which is supported by the Ministry of Health and Welfare. All samples from the National Biobank of Korea were obtained with informed consent under institutional review board-approved protocols. 


\section{References}

1. Ferlay J, Shin HR, Bray F, Forman D, Mathers C, Parkin DM. Estimates of worldwide burden of cancer in 2008: GLOBOCAN 2008. Int J Cancer 2010;127:2893-2917.

2. Herzog CE, Andrassy RJ, Eftekhari F. Childhood cancers: hepatoblastoma. Oncologist 2000;5:445-453.

3. Bruix J, Sherman M. Management of hepatocellular carcinoma: an update. Hepatology 2011;53:1020-1022.

4. Llovet JM, Ricci S, Mazzaferro V, Hilgard P, Gane E, Blanc JF, et al. Sorafenib in advanced hepatocellular carcinoma. N Engl J Med 2008; 359:378-390.

5. Cheng AL, Kang YK, Chen Z, Tsao CJ, Qin S, Kim JS, et al. Efficacy and safety of sorafenib in patients in the Asia-Pacific region with advanced hepatocellular carcinoma: a phase III randomised, doubleblind, placebo-controlled trial. Lancet Oncol 2009;10:25-34.

6. Sivaprakasam P, Gupta AA, Greenberg ML, Capra M, Nathan PC. Survival and long-term outcomes in children with hepatoblastoma treated with continuous infusion of cisplatin and doxorubicin. J Pediatr Hematol Oncol 2011;33:e226-e230.

7. Vander Heiden MG. Targeting cancer metabolism: a therapeutic window opens. Nat Rev Drug Discov 2011;10:671-684.

8. Dang CV. MYC, metabolism, cell growth, and tumorigenesis. Cold Spring Harb Perspect Med 2013;3:a014217.

9. DeBerardinis RJ, Lum JJ, Hatzivassiliou G, Thompson CB. The biology of cancer: metabolic reprogramming fuels cell growth and proliferation. Cell Metab 2008;7:11-20.

10. DeBerardinis RJ, Cheng T. Q's next: the diverse functions of glutamine in metabolism, cell biology and cancer. Oncogene 2010;29:313-324.

11. DeBerardinis RJ, Mancuso A, Daikhin E, Nissim I, Yudkoff M, Wehrli S, Thompson CB. Beyond aerobic glycolysis: transformed cells can engage in glutamine metabolism that exceeds the requirement for protein and nucleotide synthesis. Proc Natl Acad Sci U S A 2007;104:19345-19350.

12. Shanware NP, Mullen AR, DeBerardinis RJ, Abraham RT. Glutamine: pleiotropic roles in tumor growth and stress resistance. J Mol Med (Berl) 2011;89:229-236.

13. Qing G, Li B, Vu A, Skuli N, Walton ZE, Liu X, et al. ATF4 regulates MYC-mediated neuroblastoma cell death upon glutamine deprivation. Cancer Cell 2012;22:631-644.

14. Taylor L, Curthoys NP. Glutamine metabolism: role in acid-base balance. Biochem Mol Biol Educ 2004;32:291-304.
15. Baek SH, Kim KI. Emerging roles of orphan nuclear receptors in cancer. Annu Rev Physiol 2014;76:177-195.

16. Pearen MA, Muscat GE. Orphan nuclear receptors and the regulation of nutrient metabolism: understanding obesity. Physiology (Bethesda) 2012;27:156-166.

17. Jetten AM. Retinoid-related orphan receptors (RORs): critical roles in development, immunity, circadian rhythm, and cellular metabolism. Nucl Recept Signal 2009;7:e003.

18. Kim H, Lee JM, Lee G, Bhin J, Oh SK, Kim K, et al. DNA damageinduced RORalpha is crucial for p53 stabilization and increased apoptosis. Mol Cell 2011;44:797-810.

19. Lee JM, Kim IS, Kim H, Lee JS, Kim K, Yim HY, et al. RORalpha attenuates Wnt/beta-catenin signaling by PKCalpha-dependent phosphorylation in colon cancer. Mol Cell 2010;37:183-195.

20. Contractor T, Harris CR. p 53 negatively regulates transcription of the pyruvate dehydrogenase kinase Pdk2. Cancer Res 2012;72:560-567.

21. Steinman RA, Huang J, Yaroslavskiy B, Goff JP, Ball ED, Nguyen A. Regulation of p21(WAF1) expression during normal myeloid differentiation. Blood 1998;91:4531-4542.

22. Park KK, Deok Ahn J, Lee IK, Magae J, Heintz NH, Kwak JY, et al. Inhibitory effects of novel E2F decoy oligodeoxynucleotides on mesangial cell proliferation by coexpression of E2F/DP. Biochem Biophys Res Commun 2003;308:689-697.

23. Yuneva MO, Fan TW, Allen TD, Higashi RM, Ferraris DV, Tsukamoto T, et al. The metabolic profile of tumors depends on both the responsible genetic lesion and tissue type. Cell Metab 2012;15:157170.

24. Vander Heiden MG, Cantley LC, Thompson CB. Understanding the Warburg effect: the metabolic requirements of cell proliferation. Science 2009;324:1029-1033.

25. Schulze A, Harris AL. How cancer metabolism is tuned for proliferation and vulnerable to disruption. Nature 2012;491:364-373.

26. Son J, Lyssiotis CA, Ying H, Wang X, Hua S, Ligorio M, et al. Glutamine supports pancreatic cancer growth through a KRAS-regulated metabolic pathway. Nature 2013;496:101-105.

\section{Supporting Information}

Additional Supporting Information may be found at onlinelibrary.wiley.com/doi/10.1002/hep.27577/suppinfo. 